\title{
Large area all-optical ultrasound imaging using robotic control
}

Richard J. Colchester, Erwin J. Alles, George Dwyer, Efthymios Maneas, Danail Stoyanov, et al.

Richard J. Colchester, Erwin J. Alles, George Dwyer, Efthymios Maneas, Danail Stoyanov, Adrien E. Desjardins, "Large area all-optical ultrasound imaging using robotic control," Proc. SPIE 11077, Opto-Acoustic Methods and Applications in Biophotonics IV, 110771G (19 July 2019); doi: 10.1117/12.2525379

SPIE. Event: European Conferences on Biomedical Optics, 2019, Munich, Germany 


\title{
Large Area All-Optical Ultrasound Imaging Using Robotic Control
}

\author{
Richard J. Colchester ${ }^{\mathrm{a}, \mathrm{b}}$, Erwin J. Alles ${ }^{\mathrm{a}, \mathrm{b}}$, George Dwyer ${ }^{\mathrm{a}, \mathrm{b}, \mathrm{c}}$, Efthymios Maneas ${ }^{\mathrm{a}, \mathrm{b}}$, Danail \\ Stoyanov ${ }^{\mathrm{b}, \mathrm{c}, \mathrm{d}}$, and Adrien E. Desjardins ${ }^{\mathrm{a}, \mathrm{b}}$ \\ aDepartment of Medical Physics and Biomedical Engineering, University College London, \\ London, UK \\ ${ }^{\mathrm{b}}$ Wellcome/EPSRC Centre for Interventional and Surgical Sciences, University College \\ London, London, UK \\ ${ }^{\mathrm{C}}$ UCL Robotics Institute, University College London, London, UK \\ ${ }^{\mathrm{d}}$ Department of Computer Science, University College London, London, UK
}

\begin{abstract}
In this study we report the integration of an all-optical ultrasound probe and robotic manipulator. The alloptical ultrasound probe comprised two optical fibres, a MWCNT/PDMS composite coated multimode fibre for ultrasound generation, and a concave Fabry-Pérot fibre optic hydrophone for ultrasound reception. The ultrasound probe generated pressures in excess of $2 \mathrm{MPa}$ at $1.5 \mathrm{~mm}$, with a corresponding $-6 \mathrm{~dB}$ bandwidth of ca. $30 \mathrm{MHz}$. The probe was built into a rigid endoscope (outer diameter: $5 \mathrm{~mm}$, length: $300 \mathrm{~mm}$ ) and mounted on a robotic manipulator. Ultrasound A-lines were acquired during robot manipulation in order to reconstruct a 3D image which was displayed as a point cloud. Large area images $(80 \times 80 \mathrm{~mm})$ of a tissue mimicking gel wax phantom where acquired and displayed in real-time. This work demonstrates the potential for integrating miniature fibre optic ultrasound devices with robotics.
\end{abstract}

Keywords: All-Optical Ultrasound, Laser-Generated Ultrasound, Ultrasound, Robotics

\section{INTRODUCTION}

High resolution imaging from miniaturised devices is desirable for guiding minimally invasive surgical procedures. All-optical ultrasound (OpUS) is emerging as an imaging paradigm well-suited for these applications. Here, ultrasound is both generated and received using light. For ultrasound generation pulsed or modulated light is absorbed by an optically absorbing material; the subsequent heating of the material leads to a pressure rise which propagates as an ultrasound wave. ${ }^{1}$ For optical ultrasound reception interferometric methods can be used, here the impinging ultrasound waves cause deformations in resonant structures that are optically monitored. ${ }^{2}$ These optical methods can provide high sensitivity and broad ultrasound bandwidths, as well as immunity to electromagnetic interference. ${ }^{3,4}$

The use of optical fibres in OpUS probes has enabled highly miniaturised ultrasound imaging devices capable of $2 \mathrm{D}^{3}$ and $3 \mathrm{D}$ imaging. ${ }^{2,5}$ In a recent study one such device was integrated into a needle to provide real-time M-mode imaging during an in vivo experiment. ${ }^{4}$ Further, real-time rotational imaging using optical fibres has been demonstrated on ex vivo swine carotid arteries. ${ }^{6}$ Additionally, the combination of all-optical robotics has demonstrated the potential for its use to provide feedback and improve robotic control. ${ }^{7}$

In this study we combined a robotic manipulator with a fibre optic ultrasound probe, which comprised a nanocomposite coated multimode fibre for ultrasound generation and a concave Fabry-Pérot fibre optic hydrophone for ultrasound reception. The ultrasound probe was built into a custom rigid endoscope and a custom computer interface was used to acquire and display ultrasound A-lines in real-time during robot manipulation. Using pre-planned robotic trajectories, 3D imaging of a tissue mimicking phantom was carried out.

Further author information: (Send correspondence to Richard J. Colchester)

Richard J. Colchester: E-mail: richard.colchester@ucl.ac.uk

Opto-Acoustic Methods and Applications in Biophotonics IV, edited by Vasilis Ntziachristos,

Roger Zemp, Proc. of SPIE-OSA Vol. 11077, 110771G · C 2019 SPIE-OSA

CCC code: $1605-7422 / 19 / \$ 21 \cdot$ doi: $10.1117 / 12.2525379$

Proc. of SPIE-OSA Vol. 11077 110771G-1 

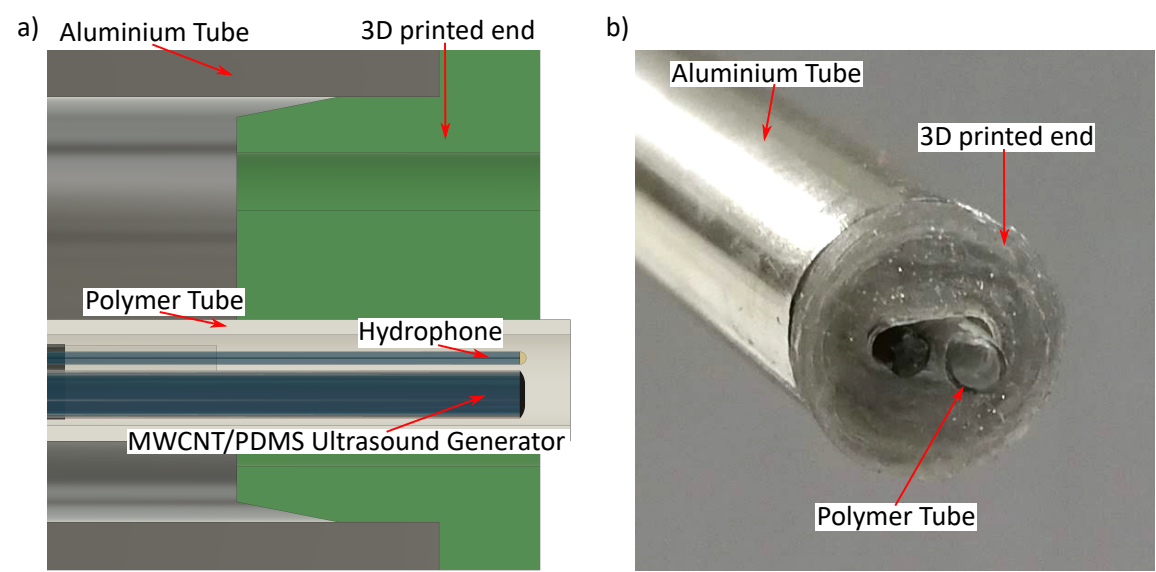

Figure 1. a) Schematic cross-section of the rigid endoscope tip, with a fibre optic all-optical ultrasound probe contained within a polymer tube. b) Photograph of the endoscope tip showing the aluminium tube, the 3D printed end cap, and the polymer tubing containing the ultrasound transmitting and receiving optical fibres.

\section{DEVICE DESIGN AND OPERATION}

\subsection{All-optical ultrasound probe}

The OpUS probe comprised two optical fibres: a $400 \mu \mathrm{m}$ core diameter multimode fibre coated with a multi-walled carbon nanotube (MWCNT) and polydimethylsiloxane (PDMS) composite coating to generate ultrasound, ${ }^{5}$ and a single mode (SMF-28) fibre coated with a concave Fabry-Pérot hydrophone to receive ultrasound. ${ }^{2}$ The two fibres were held adjacent, with the distal end surfaces aligned, using heat shrink tubing and then inserted into polymethylpentene tubing for protection, resulting in an imaging probe diameter of just $1.2 \mathrm{~mm}$ (Figure 1). Ultrasound was generated photoacoustically using a Q-switched Nd:YAG laser (DSS-1064 Q, Crylas, Germany) with a pulse energy of $20 \mu \mathrm{J}$, a pulse width of $1.6 \mathrm{~ns}$, and a wavelength of $1064 \mathrm{~nm}$. The Fabry-Pérot fibre optic hydrophone was interrogated using a continuous wave tunable laser (TSL-550, Santec, UK) via a circulator. The reflected optical signal was measured using a photodiode. The low frequency component of the reflected optical signal was used to track the optimum bias point of the sensor. The high frequency component of the reflection, which was encoded with the received ultrasound, was digitised at $62.5 \mathrm{MS} / \mathrm{s}$ (M4i.4420-x8, Spectrum, Germany). The optical ultrasound probe generated pressures $>2 \mathrm{MPa}$ at a distance of $1.5 \mathrm{~mm}$, with a corresponding $-6 \mathrm{~dB}$ bandwidth of $c a .30 \mathrm{MHz}$. The axial resolution of the device was $c a .60 \mu \mathrm{m} .{ }^{4}$ The acquired ultrasound A-lines were band-pass filtered (Butterworth, $4^{\text {th }}$ order, $3-25 \mathrm{MHz}$ ) to reduce the noise, prior to cross-talk removal. ${ }^{3}$ Subsequently the signal envelope was found using a Hilbert transform and then a log transform was applied for display. The maximum values along each A-scan were extracted, and displayed as a point cloud for 3D viewing.

\subsection{Endoscope design}

The rigid endoscope comprised a stainless steel tube (outer diameter: $5 \mathrm{~mm}$, length: $300 \mathrm{~mm}$ ) with a custom 3D printed end cap. The 3D printed end cap had an eccentric hole, with space to fit a $1.3 \mathrm{~mm}$ diameter device (working channel) next to a $1.7 \mathrm{~mm}$ diameter device (ultrasound probe) (Figure 1). The endoscope was fixed to the robotic manipulator (KUKA LBR iiwa 14 R820, KUKA AG, Germany) using a custom 3D printed mount.

\subsection{Interface and control}

The robotic manipulator was controlled using the Fast Robotic Interface, implemented as a Robotic Operating System (ROS) hardware interface for controlling the joint positions. ${ }^{8}$ This allowed all joint positions, velocity, and effort limits to be set and enforced prior to being sent to the controller. The Cartesian control and planning was handled using the Moveit motion planning framework, ${ }^{9}$ using the TRAC-IK plugin ${ }^{10}$ and the open motion planning library ${ }^{11}$ as the inverse kinematics solver and path planner, respectively. The acquired ultrasound A-lines were streamed to the robotic control computer over a ROS interface using a custom message type. Thresholded values of the ultrasound A-lines were plotted within a 3D volume in real-time using the position 

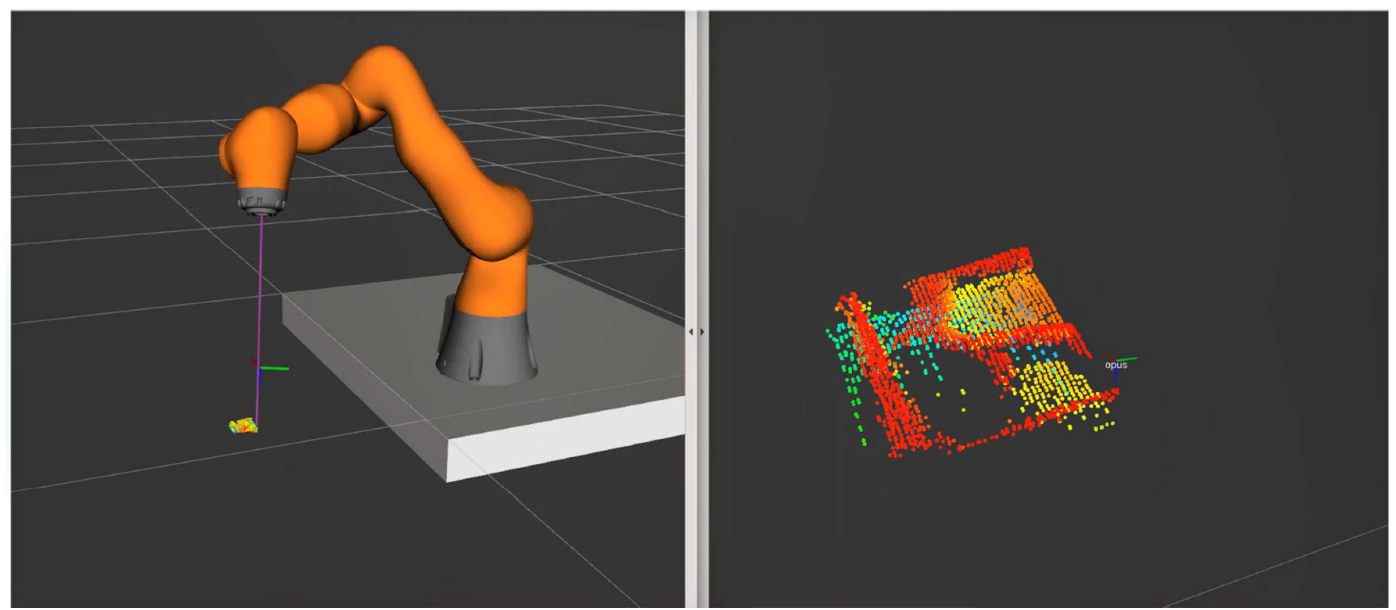

Figure 2. Screen grab of the visualisation of the robotic manipulator (left) and acquired ultrasound point cloud (right), as displayed during data acquisition.

and orientation of the endoscope tip, as found using forward kinematics from the manipulator (Figure 2). The raw and processed data was also saved to allow additional offline plotting using MATLAB.

\section{EXPERIMENTS AND RESULTS}

To demonstrate the potential of this robotic integration, a gel wax tissue mimicking phantom ${ }^{12}$ was imaged (Figure 3). The phantom was based on vascular structures from a human placenta, extracted using 3D drawing software and converted to a 3D printed mould. Molten gel wax with solid glass spheres incorporated to was poured into the mould to form the tissue mimicking phantom. During scans a remote centre of motion (RCM) was set a fixed distance $(160 \mathrm{~mm})$ above the placenta phantom. This constrained the endoscope to pivot around this point, such that it did move laterally, a key feature for application to minimally invasive scenarios, where the endoscope must be stationary at the access point.

Two scan patterns were used; a square raster scan $(80 \times 80 \mathrm{~mm}$, step size: $1 \mathrm{~mm}$, scan time: 43 minutes $)$, and a spiral scan $(80 \mathrm{~mm}$ diameter, 40 cycles, scan time: 45 minutes). At each position in the scan the robotic manipulator paused for $0.1 \mathrm{~s}$ to ensure synchronicity. The resulting images show the surface vessels across the entire imaged region (Figure 4). Additionally, back-scatter is visible through the first few millimetres. Signal descending below the placenta phantom was visible due to strong reflections occurring away from the endoscope direction originating from highly echogenic metal posts used to secure the phantom during the experiment. These data sets demonstrate the potential for OpUS to acquire large 3D images.

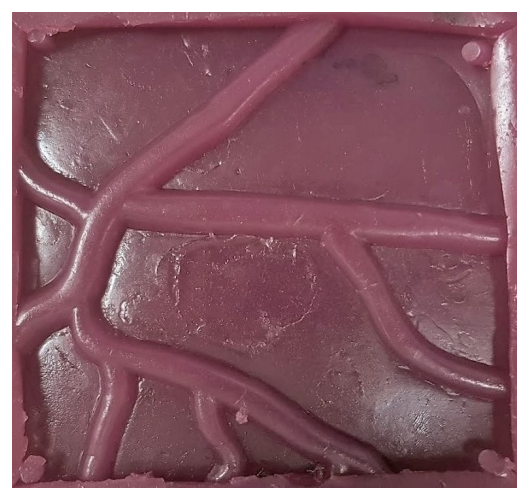

Figure 3. Top down photograph of the tissue mimicking gel wax placenta phantom. 

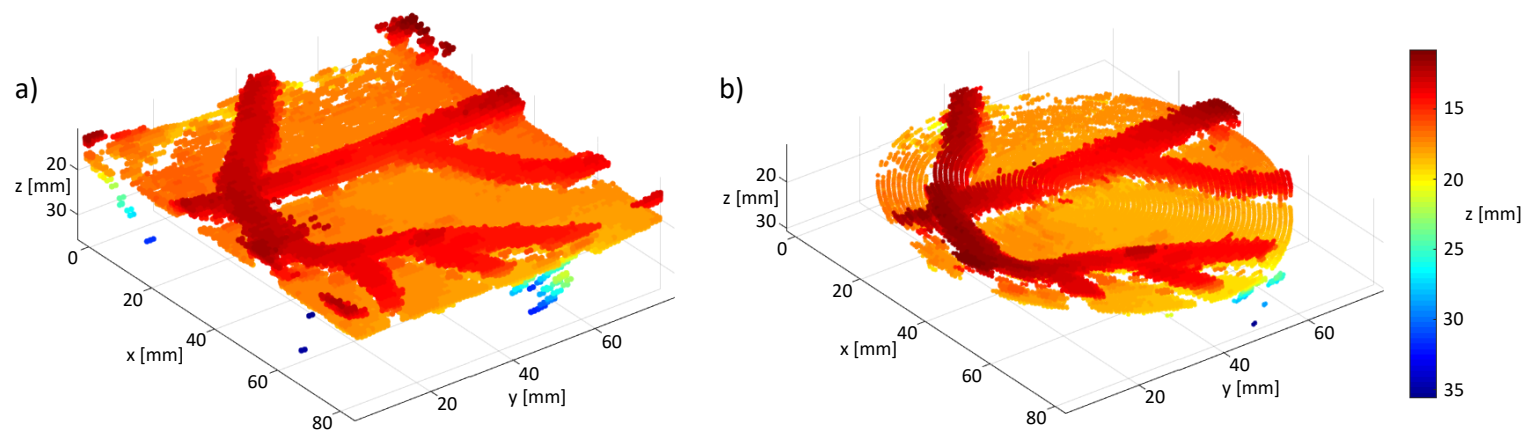

Figure 4. Ultrasound point clouds of a gel wax placenta phantom acquired using: a) a raster scan pattern, and b) a spiral scan pattern. Colour map corresponds to image depth (z).

\section{CONCLUSION}

In this work we demonstrate the integration of OpUS with a robotic manipulator. OpUS imaging provided A-lines with an imaging depth of $30 \mathrm{~mm}$ and an axial resolution of $60 \mu \mathrm{m}$. The OpUS probe used was integrated into an endoscope with space for a separate working channel, and a phantom was imaged. In future experiments the working channel may be used to house surgical tools, such as an optical fibre to provide laser ablation. Additionally, the OpUS probe can be optimised to provide improved lateral resolution. The divergence of the generated ultrasound beam could be reduced by using a concave ultrasound generating surface ${ }^{13}$ or larger diameter planar surfaces,${ }^{14}$ to achieve a focus within tissue.

One advantage of the OpUS modality is the ability to integrate complementary modalities by tailoring the optical absorption profile of the ultrasound generating surface. ${ }^{15}$ Previously this has been used to demonstrate co-registered ultrasound and photoacoustic imaging, but could be easily tailored to provide laser ablation through the same optical fibre as ultrasound generation.

For the current acquisition, a pause in the robotic manipulator motion was required to allow ultrasound data to be synchronised with endoscope tip position. This resulted in an acquisition rate of approximately 2.5 positions per second. However, the current system allows for $60 \mathrm{~A}$-lines per second to be transferred and saved, thus in future work the acquisition speed will be improved by synchronising the computers used. This would allow for a ten-fold improvement in acquisition time.

The presented ultrasound images demonstrate the potential for large area $(80 \times 80 \mathrm{~mm})$ OpUS imaging with robotic manipulation. In the context of surgery, these images would allow for depth ranging and imaging through murky environments, such as amniotic fluid. Further, additional processing would enable visualisation and identification of subsurface structures, such as vessels. The combination with robotics may ultimately lead to automation and feedback during procedures.

\section{REFERENCES}

[1] Cox, B. T. and Beard, P. C., "Fast calculation of pulsed photoacoustic fields in fluids using k -space methods," The Journal of the Acoustical Society of America 117(6), 3616-3627 (2005).

[2] Guggenheim, J. A., Li, J., Allen, T. J., Colchester, R. J., Noimark, S., Ogunlade, O., Parkin, I. P., Papakonstantinou, I., Desjardins, A. E., Zhang, E. Z., and Beard, P. C., "Ultrasensitive plano-concave optical microresonators for ultrasound sensing," Nature Photonics 11(11), 714-719 (2017).

[3] Colchester, R. J., Zhang, E. Z., Mosse, C. A., Beard, P. C., Papakonstantinou, I., and Desjardins, A. E., "Broadband miniature optical ultrasound probe for high resolution vascular tissue imaging," Biomedical Optics Express 6(4), 1502-1511 (2015).

[4] Finlay, M. C., Mosse, C. A., Colchester, R. J., Noimark, S., Zhang, E. Z., Ourselin, S., Beard, P. C., Schilling, R. J., Parkin, I. P., Papakonstantinou, I., and Desjardins, A. E., "Through-needle all-optical ultrasound imaging in vivo: a preclinical swine study," Light: Science 83 Applications 6(12), e17103 (2017). 
[5] Noimark, S., Colchester, R. R. J., Blackburn, B. B. J., Zhang, E. E. Z., Alles, E. J. E., Ourselin, S., Beard, P. C. P., Papakonstantinou, I., Parkin, I. I. P., and Desjardins, A. A. E., "Carbon-Nanotube-PDMS Composite Coatings on Optical Fibers for All-Optical Ultrasound Imaging," Advanced Functional Materials 26(46), 8390-8396 (2016).

[6] Colchester, R. J., Little, C., Dwyer, G., Noimark, S., Alles, E. J., Zhang, E. Z., Loder, C. D., Parkin, I. P., Papakonstantinou, I., Beard, P. C., Finlay, M. C., Rakhit, R. D., and Desjardins, A. E., "All-Optical Rotational Ultrasound Imaging," Scientific Reports 9(1), 5576 (2019).

[7] Gruijthuijsen, C., Colchester, R., Devreker, A., Javaux, A., Maneas, E., Noimark, S., Xia, W., Stoyanov, D., Reynaerts, D., Deprest, J., Ourselin, S., Desjardins, A., Vercauteren, T., and Poorten, E. V., "Haptic Guidance Based on All-Optical Ultrasound Distance Sensing for Safer Minimally Invasive Fetal Surgery," J. Med. Robot. Res., 1841001 (2018).

[8] Chitta, S., Marder-Eppstein, E., Meeussen, W., Pradeep, V., Rodríguez Tsouroukdissian, A., Bohren, J., Coleman, D., Magyar, B., Raiola, G., Lüdtke, M., and Fernandez Perdomo, E., "ros_control: A generic and simple control framework for ROS," J. Open Source Softw. 2(20), 456 (2017).

[9] Chitta, S. and Sucan, I. A., "Moveit!." Available at http://moveit.ros.org/.

[10] Beeson, P. and Ames, B., " $\{$ TRAC-IK $\}$ : An Open-Source Library for Improved Solving of Generic Inverse Kinematics," in [Proc. IEEE RAS Humanoids Conf.], (2015).

[11] Şucan, I. A., Moll, M., and Kavraki, L. E., "The Open Motion Planning Library," IEEE Robotics \&3 Automation Magazine 19(4), 72-82 (2012). http://ompl.kavrakilab.org.

[12] Maneas, E., Xia, W., Nikitichev, D. I., Daher, B., Manimaran, M., Wong, R. Y. J., Chang, C.-W., Rahmani, B., Capelli, C., Schievano, S., Burriesci, G., Ourselin, S., David, A. L., Finlay, M. C., West, S. J., Vercauteren, T., and Desjardins, A. E., "Anatomically realistic ultrasound phantoms using gel wax with 3D printed moulds," Phys. Med. Biol. 63(1), 015033 (2018).

[13] Alles, E. J., Noimark, S., Zhang, E., Beard, P. C., and Desjardins, A. E., "Pencil beam all-optical ultrasound imaging," Biomedical Optics Express 7(9), 3696 (2016).

[14] Colchester, R. J., Alles, E. J., and Desjardins, A. E., "A directional fibre optic ultrasound transmitter based on a reduced graphene oxide and polydimethylsiloxane composite," Applied Physics Letters 114(11), 113505 (2019).

[15] Noimark, S., Colchester, R. J., Poduval, R. K., Maneas, E., Alles, E. J., Zhao, T., Zhang, E. Z., Ashworth, M., Tsolaki, E., Chester, A. H., Latif, N., Bertazzo, S., David, A. L., Ourselin, S., Beard, P. C., Parkin, I. P., Papakonstantinou, I., and Desjardins, A. E., "Polydimethylsiloxane Composites for Optical Ultrasound Generation and Multimodality Imaging," Advanced Functional Materials 28(9), 1704919 (2018). 